\title{
7. Vocabulary
}

In COBOL, words fall into two types:

1. words that have a special meaning - e.g. 'DISPLAY', 'ACCEPT', 'DIVISION' - these are called Reserved Words and may only be used for the purposes stated in the COBOL manual (refer to lesson 7a for list);

2. words that have no special meaning in COBOL but are invented by the programmer as names for items of data, paragraphs, etc - these are called programmer-defined words).

Any name the programmer invents for data items, paragraphs, etc:

a) may be up to 30 characters long;

b) may contain any of the characters ' $\mathrm{A}$ ' to ' $\mathrm{Z}$ ', 'a' to ' $z$ ', 'O' to '9' and '-' but must not begin or end with a '-' and must not (with some exceptions) consist solely of numbers;

c) must not contain any spaces.

It is usual to make up names that have a clear meaning:

e.g. EMPLOYEE-NAME, STUDENT-MATHS-MARK would be clear names for items of data, while CALCULATE-PAY and FIND-AVERAGE-MARK would be clear names for paragraphs (sections of the program).

Exercise

1. Which of the following are allowed as programmer-defined words? For each word that is not valid say why not.

1. TOTAL-PAY

2. WORK-OUT-TAX

3. GET-STAFF-DETAILS

4. EMPLOYEE-NAME

5. STUDENT-TIMETABLE

6. DISPLAY

7. CALCULATE*INTEREST

8. NEW EMPLOYEE NAME

9. HOURLY-RATE

10. DIVISION
11. NEW-STAFF-DETAILS

12. INTEREST

13. 2ND-STUDENT-MARK

14. TOTAL-COST

15. AVERAGE MARK

16. STOP

17. PIC

18. IDENTIFICATION

19. DATA

20. WORKING-STORAGE

2. Looking at the example programs on lessons 5 and 6:

a) list all the reserved words;

b) list all the programmer-defined words. 